\title{
Fast Global Minimization of the Active Contour/Snake Model
}

\author{
Xavier Bresson • Selim Esedoḡlu • \\ Pierre Vandergheynst • Jean-Philippe Thiran · \\ Stanley Osher
}

Published online: 14 July 2007

(C) Springer Science+Business Media, LLC 2007

\begin{abstract}
The active contour/snake model is one of the most successful variational models in image segmentation. It consists of evolving a contour in images toward the boundaries of objects. Its success is based on strong mathematical properties and efficient numerical schemes based on the level set method. The only drawback of this model is the existence of local minima in the active contour energy, which makes the initial guess critical to get satisfactory results. In this paper, we propose to solve this problem by determining a global minimum of the active contour model. Our approach is based on the unification of image segmentation and image denoising tasks into a global minimization framework. More precisely, we propose to unify three well-known image variational models, namely the snake model, the RudinOsher-Fatemi denoising model and the Mumford-Shah segmentation model. We will establish theorems with proofs to determine the existence of a global minimum of the active contour model. From a numerical point of view, we propose a new practical way to solve the active contour propagation problem toward object boundaries through a dual formulation of the minimization problem. The dual formulation,
\end{abstract}

Research supported by NIH U54RR021813, NSF DMS-0312222, NSF ACI-0321917 and NSF DMI-0327077.

X. Bresson $(\bowtie) \cdot$ P. Vandergheynst · J.-P. Thiran

Signal Processing Institute, Swiss Federal Institute of Technology

(EPFL), Lausanne, Switzerland

e-mail: xavier.bresson@epfl.ch

S. Esedoḡlu

Department of Mathematics, University of Michigan, Ann Arbor, MI, USA

S. Osher

Department of Mathematics, University of California,

Los Angeles, CA, USA easy to implement, allows us a fast global minimization of the snake energy. It avoids the usual drawback in the level set approach that consists of initializing the active contour in a distance function and re-initializing it periodically during the evolution, which is time-consuming. We apply our segmentation algorithms on synthetic and real-world images, such as texture images and medical images, to emphasize the performances of our model compared with other segmentation models.

Keywords Active contour - Global minimization . Weighted total variation norm $\cdot$ ROF model . Mumford-Shah energy $\cdot$ Dual formulation of TV

\section{Introduction and Motivations}

The image segmentation problem is fundamental in the field of computer vision. It is a core component toward e.g. automated vision systems and medical applications. Its aim is to find a partition of an image into a finite number of semantically important regions. Various variational and partial differential equations (PDEs)-based methods have been proposed to extract objects of interest in images such as the well-known and successful active contour/snake model, initially proposed by Kass, Witkin and Terzopoulos in [1]. The number of applications of this method is numerous in various image processing applications such as in medical imaging to extract anatomical structures [2-4].

Following the first model of active contours, Caselles, Kimmel and Sapiro in [5] and Kichenassamy, Kumar, Olver, Tannenbaum and Yezzi in [6] proposed a new enhanced version of the snake model called the geodesic/geometric active 
contour (GAC) model. This new formulation is said geometrically intrinsic because the proposed snake energy is invariant with respect to (w.r.t.) the curve parametrization. The model is defined by the following minimization problem:

$\min _{C}\left\{E_{\mathrm{GAC}}(C)=\int_{0}^{L(C)} g\left(\left|\nabla I_{0}(C(s))\right|\right) d s\right\}$

where $d s$ is the Euclidean element of length and $L(C)$ is the length of the curve $C$ defined by $L(C)=\int_{0}^{L(C)} d s$. Hence, the energy functional (1) is actually a new length obtained by weighting the Euclidean element of length $d s$ by the function $g$ which contains information concerning the boundaries of objects [5]. The function $g$ is an edge indicator function that vanishes at object boundaries such as $g\left(\left|\nabla I_{0}\right|\right)=\frac{1}{1+\beta\left|\nabla I_{0}\right|^{2}},{ }^{1}$ where $I_{0}$ is the original image and $\beta$ is an arbitrary positive constant. The calculus of variations provides us the Euler-Lagrange equation of the functional $E_{\mathrm{GAC}}$ and the gradient descent method gives us the flow that minimizes as fast as possible $E_{\mathrm{GAC}}$ (see [5]):

$\partial_{t} C=(\kappa g-\langle\nabla g, \mathcal{N}\rangle) \mathcal{N}$,

where $\partial_{t} C:=\partial C / \partial t, t$ being an artificial time parameter, and $\kappa, \mathcal{N}$ are respectively the curvature and the normal to the curve $C$. The evolution equation of active contours, defined in (2), is well-defined because a unique viscosity solution $[5,7]$ associated to the PDE (2) exists. Osher and Sethian introduced in [8] the level set method to efficiently solve the contour propagation problem and to deal with topological changes. Equation (2) can be written in the level set form as follows:

$\partial_{t} \phi=\left(\kappa g+\left\langle\nabla g, \frac{\nabla \phi}{|\nabla \phi|}\right\rangle\right)|\nabla \phi|$,

where $\phi$ is the level set function embedding the evolving active contour $C$ such that $C(t)=\left\{x \in \mathbf{R}^{N} \mid \phi(x, t)=0\right\}$. The PDE (3) is implemented with numerical schemes based on hyperbolic conservation laws, see Osher-Sethian [8-10], which can be highly accurate $[11,12]$ to give very fine/subpixel segmentations.

Despite the many good numerical results obtained with this segmentation model and strong theoretical properties, the snake/GAC model is highly sensitive to the initial condition. Actually, the quality of the segmentation result depends a lot on the choice of the initial contour, which means that a bad initial contour can give an unsatisfactory result. The problem of a good initial condition is related to the non-convexity of the energy functional, $E_{\mathrm{GAC}}$, to be minimized and then the existence of local minima. This drawback is not specific to this variational model because it is a

\footnotetext{
${ }^{1}$ Throughout the paper, function $g$ will denote the edge detector function.
}

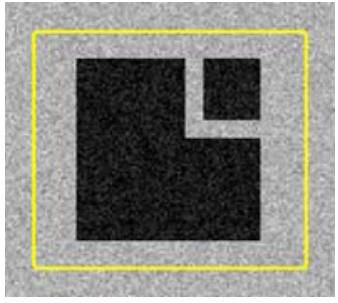

(a) Initial Snake/GAC

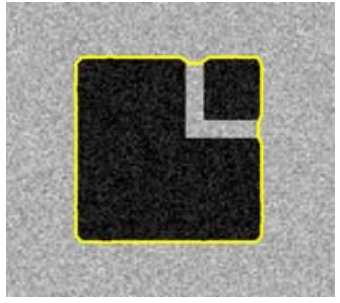

(b) Final Snake/GAC.
Fig. 1 The standard snake model, defined in (1-3), fails to segment the two objects

widespread issue when dealing with variational models in image processing which also suffer from local minima. In the case of the GAC model, the existence of local minima in $E_{\mathrm{GAC}}$ can prevent the segmentation of meaningful objects lying in images. A simple example is given in Fig. 1. The initial GAC (embedded in a level set function in order to allow natural topology changes) in Fig. 1(a) can not fully segment the two objects, Fig. 1(b), because it gets stuck in a bad local minimum. The best segmentation result should provide the two objects, which corresponds to another local minimum of $E_{\mathrm{GAC}}$ obtained with a different initial condition. Hence we wish to define an image segmentation model providing the correct result independently of the initial condition, which means that we look for a global minimum of a convex functional. We notice that the global minimum for the GAC/snake model corresponds to a point, which has no practical sense for the image segmentation task. We thus propose in this paper to define new active contour energies based on the GAC model and whose global minimum corresponds to the expected segmentation result. Hence, throughout the paper, "global minimum of the active contour model" will refer to the global minimization of new active contour models based partially on the GAC model but not to the global minimization of the GAC model, which has no sense as we previously said.

In a recent work, Chan, Esedoḡlu and Nikolova [13] proposed a new approach to deal with global minimum and overcome the limitation of local minima. In their paper, they related image segmentation to image denoising in order to find global minimizers of two denoising and segmentation models. The first model is a binary image denoising model which removes the geometric noise in a given shape and the second model is the active contours without edges (ACWE) model of Chan and Vese [14].

In this work, we develop three theoretical global minimization models for the active contour model inspired by [13]. The first model is based on the standard snake/GAC segmentation model $[1,5,6]$ and the well-known image denoising model of Rudin, Osher and Fatemi (ROF) defined in [15]. We remind that image denoising aims at removing noise in images while keeping main features such as edges 
and textures. It is interesting to notice that a unified approach of image segmentation and image denoising provides us with a global minimization solution for the active contour model subject to an intensity homogeneity constraint. Then the second model is based on the standard active contour model $[1,5,6]$ and the piecewise-constant Mumford and Shah's model [16], which is related to the ACWE model of Chan and Vese [14]. Our model will "reconcile" the classical GAC model, based on the detection of edges, and the ACWE model, based on the detection of homogeneous regions, in a single framework to globally minimize the active contour model subject to intensity homogeneity constraints. Finally, the third model uses the piecewise-smooth approximation of the Mumford-Shah model to find a global active contour subject to smooth intensity constraints.

This paper, besides developing new theoretical models to carry out the global minimization of the active contour model, also proposes new numerical schemes to perform the contour evolution in an efficient and fast way. Thus, the traditional contour propagation problem is solved with a dual formulation of the total variation (TV) norm introduced and developed in [17-21]. These original implementation schemes are easy to implement and very fast compared with usual schemes, based on the level set approach such as (3). Indeed, standard contour tracking algorithms use a distance function (DF), as a level set function, to implicitly and intrinsically represent the active contour. The main problem is that the DF is not a solution of (3), which means that the level set function does not remain a DF during the contour evolution process. This requires the user to periodically re-initialize the level set function as a DF, which is timeconsuming, to ensure correct numerical computations of the curvature and the normal to the contour. Finally, the initial active contour has also to be embedded in a DF in the standard approach, which also requires special techniques. Thus the main contributions of this paper are as follows:

(1) Introduction of three theoretical models to carry out the global minimization of the active contour segmentation model based on the GAC model, the Rudin-OsherFatemi denoising model and the Mumford-Shah model.

(2) Definition of an enhanced segmentation model by unifying into a global minimization framework the complementary approaches of the geodesic/geometric active contours model, based on the detection of edge points, and the active contours without edges model, based on the detection of homogeneous regions.

(3) Presentation of new numerical schemes, based on the dual formulation of the TV-norm, to solve the global minimization problem of the snake propagation in an efficient, easy and fast way.

The next section defines the global minimization model based on the snake method and the ROF model, which pro- vides a unified way to perform image segmentation and image denoising. Then Sect. 3 introduces the second global minimization model based on the piecewise-constant approximation of the Mumford-Shah model, which is known as the ACWE model of Chan-Vese. We show that our model improves the performances of the ACWE. Section 4 presents the global minimization model based on the piecewisesmooth approximation of the Mumford-Shah model. We compare the proposed model in this paper to other works in Sect. 5. Finally, we conclude in Sect. 6 and give in Appendix the proofs of the introduced theorems.

\section{Global Minimization of the Active Contour Model Based on the ROF Model}

\subsection{Theoretical Model}

In this section, we unify the snake segmentation model with the denoising Rudin, Osher and Fatemi model defined in [15]. The ROF model is one of the most famous and influential variational and PDE-based image denoising models in image processing. This denoising technique removes the noise while preserving the edges in images. The minimization problem associated with the ROF model is as follows:

$$
\min _{u}\{E_{\mathrm{ROF}}(u, \lambda)=\underbrace{\int_{\Omega}|\nabla u| d x}_{=: T V(u)}+\lambda \int_{\Omega}(u-f)^{2} d x\},
$$

where $\Omega \subset \mathbf{R}^{N}$ is an open set representing the image domain, $f$ is a given (possibly noisy) image, $T V(u)$ is the total variation norm of the function $u$, and $\lambda>0$ is an arbitrary parameter related to the scale of observation of the solution. Based on the approach of Chan, Esedog lu and Nikolova in [13], we propose the following (non-strictly) convex energy defined for any given observed image $f \in L^{1}(\Omega)$, any function $g: \Omega \rightarrow \mathbf{R}^{+}$independent of $u$ and any positive parameter $\lambda$ :

$$
E_{1}(u, \lambda):=\underbrace{\int_{\Omega} g(x)|\nabla u| d x}_{=: T V_{g}(u)}+\lambda \int_{\Omega}|u-f| d x .
$$

The differences between Energy (5) and the ROF model (4) are the introduction of the weighted $T V$-norm, $T V_{g}(u)$ with a weight function $g(x)$ and the replacement of the $L^{2}$-norm by the $L^{1}$-norm as a fidelity measure w.r.t. the given image $f$. These modifications have two important consequences. First, the $L^{1}$-norm, which has been introduced and well studied in [22-29], outperforms the standard ROF regularization model with the $L^{2}$-norm for some applications and presents important geometric properties concerning global minimizers of functionals, which will be used 
for the active contour global minimization problem. Second, the introduction of a weight function, $g$, in the TVnorm gives us the link between the snake/GAC model and the proposed functional, $E_{1}$, because the snake energy, defined in (1), is equal to the weighted TV-norm when $g$ is an edge indicator function and the function $u$ is a characteristic function, $\mathbf{1}_{\Omega_{C}}$, of a closed set $\Omega_{C} \subset \Omega$ which $C$ denotes the (non-connected) boundaries of $\Omega_{C}$ :

$$
\begin{aligned}
T V_{g}\left(u=\mathbf{1}_{\Omega_{C}}\right) & =\int_{\Omega} g(x)\left|\nabla \mathbf{1}_{\Omega_{C}}\right| d x \\
& =\int_{C} g(s) d s=E_{\mathrm{GAC}}(C) .
\end{aligned}
$$

Before establishing the global minimization theorem for the active contour model, let us develop here the comparison between the standard ROF model, the ROF model with the $L^{1}$-norm and the proposed model in (5). Chan and Esedoḡlu studied in [29] the differences between the ROF model and the ROF model that uses the $L^{1}$-norm as a fidelity measure. They showed that the $L^{1}$-norm better preserves the contrast than the $L^{2}$-norm and the order in which the features disappear, in the regularization process, is completely determined in terms of the geometry (such as area and length) of the features and not in terms of the contrast such as in the standard model. Figure 2 presents the difference between the ROF model using the $L^{1}$-norm and our model using the $L^{1}$-norm and the weight function $g$. The parameter $\lambda$ for both models in Fig. 2(a) is chosen such that the four small circles are removed while keeping the larger one. We can see that our model, using an edge indicator function, gives us a better quality result because the edge function better preserves the geometry of the original features such as the corners and the largest disk.

Besides improving the regularization process of the ROF model, Energy (5) provides a global minimization of the active contour model. The global minimization result is based on the following theorem:

Theorem 1 Suppose that $g(x) \in[0,1]$ and $f(x)$, the given image, is the characteristic function of a bounded domain $\Omega_{f} \subset \Omega$, for any given $\lambda>0$, if $u(x)$ is any minimizer of $E_{1}(., \lambda)$, then for almost every $\mu \in[0,1]$ we have that the characteristic function

$\mathbf{1}_{\Omega_{C}(\mu)=\{x: u(x)>\mu\}}(x)$,

where $C$ is the boundary of the set $\Omega_{C}$, is a global minimizer of $E_{1}(., \lambda)$.

Proof See Appendix.

Theorem 1 states the relation between the global minimization of Energy $E_{1}$ and the snake/GAC model. Indeed,

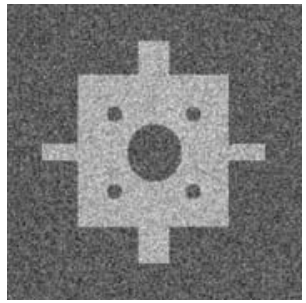

(a) Original Image $f \in$ $[0,1]$.

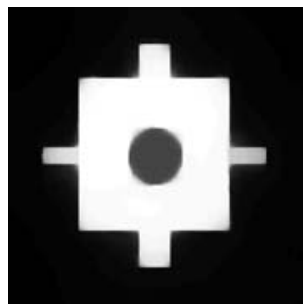

(c) Final $u$ with $T V-L^{1}$

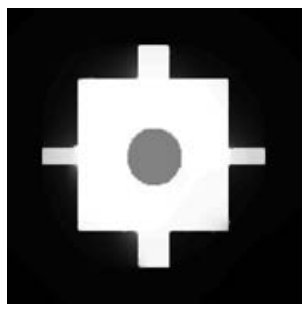

(e) Final $u$ with $E_{1}=$ $T V_{g}-L^{1}$.

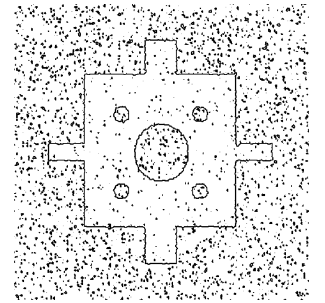

(b) Level Set $\partial\{x$ $f(x)>0.5\}$.

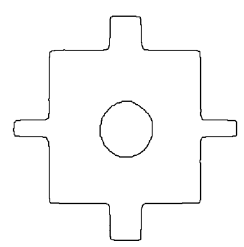

(d) Level Set $\partial\{x$ $u(x)>0.5\}$.

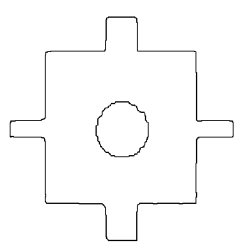

(f) Level Set $\partial\{x$ $u(x)>0.5\}$.
Fig. 2 Comparison between the ROF model using $L^{1}$-norm as a fidelity measure, (c-d), and our model using the weighted TV-norm and the $L^{1}$-norm, (e-f). The difference between both models is clear. The result generated by our model better preserves the geometry of the original features such as the corners and the largest circle

when function $u$ is a characteristic function of a set $\Omega_{C}$, whose boundary is denoted $C$, the expression of Energy $E_{1}$ is thus equal to:

$$
\begin{aligned}
E_{1}\left(u=\mathbf{1}_{\Omega_{C}}, \lambda\right) & =\int_{\Omega} g\left|\nabla \mathbf{1}_{\Omega_{C}}\right| d x+\lambda \int_{\Omega}\left|\mathbf{1}_{\Omega_{C}}-f\right| d x \\
& =\int_{C} g d s+\lambda \int_{\Omega}\left|\mathbf{1}_{\Omega_{C}}-f\right| d x .
\end{aligned}
$$

Hence, minimizing Energy (6) is equivalent to

minimize $\int_{C} g d s=E_{\mathrm{GAC}}(C)$ (the snake/GAC energy (1)),

while

approximating the given image $f$ (in the $L^{1}$ sense) by a binary function of a set/region $\Omega_{C}$.

Hence minimizing Energy $E_{1}$ corresponds to find an active contour which minimizes the GAC energy while recover- 
ing the boundary of a binary image. We underline that globally minimizing $E_{1}$ is obviously not equivalent to globally minimizing the GAC energy defined in (1). As we said in Sect. 1, it has no sense to globally minimize the GAC model because its global minimum corresponds to a point. Here, we globally minimize a new active contour energy, namely $E_{1}$, partially based on the GAC and whose global minimum corresponds to the expected segmentation result.

Finally, since Energy $E_{1}$ is convex but not strictly convex, it does not possess local minima that are not global minima. Hence any minimizer of Energy $E_{1}$ is a global minimizer. Thus, according to Theorem 1 , for any minimizer $u$ of $E_{1}$, the contour $C$ of the set $\{x: u(x)>\mu\}$ for any $\mu \in[0,1]$ is a global minimizer of the active contour energy for (possibly noisy) binary images such as the image in Fig. 1.

The next two sections define two numerical schemes to compute the global minimum of the active contour model. Section 2.2 gives a PDE to find a global minimum and Sect. 2.3 introduces a new algorithm, based on a dual formulation of the TV-norm, to quickly compute a global minimizer.

\subsection{Standard Minimization Based on a PDE}

As we previously said, any minimizer $u$ of $E_{1}$ provides a global minimum to the active contour model. Hence, the standard calculus of variation model can be used to determine a PDE which is guaranteed to find a global minimizer of the segmentation model. The minimization flow of Functional $E_{1}$ is:

$$
\begin{aligned}
\partial_{t} u & =\operatorname{div}\left(g \frac{\nabla u}{|\nabla u|}\right)+\lambda \frac{u-f}{|u-f|} \\
& =g \operatorname{div}\left(\frac{\nabla u}{|\nabla u|}\right)+\left\langle\nabla g, \frac{\nabla u}{|\nabla u|}\right\rangle+\lambda \frac{u-f}{|u-f|},
\end{aligned}
$$

where the first term of the right-hand side of (7) is the curvature of the level sets of $u, \operatorname{div}\left(\frac{\nabla u}{|\nabla u|}\right)$, multiplies by the edge indicator function $g$, the second term is a shock term which enhances the detection of edges and the third term of the right-hand side is a data fidelity term w.r.t. the observed image $f$. The evolution equation (7) can be discretized with the following explicit numerical scheme:

$$
\begin{aligned}
\frac{u^{n+1}-u^{n}}{\delta t}= & g\left(\sqrt{\left(D_{x}^{0} f\right)^{2}+\left(D_{y}^{0} f\right)^{2}}\right) \\
& \times\left\{D_{x}^{-} \mathcal{N}_{x, \varepsilon_{1}}^{+, n}+D_{y}^{-} \mathcal{N}_{y, \varepsilon_{1}}^{+, n}\right\} \\
& +\left(D_{x}^{0} g\right)_{\wedge} \cdot \mathcal{N}_{x, \varepsilon_{1}}^{-, n}+\left(D_{x}^{0} g\right)_{\vee} \cdot \mathcal{N}_{x, \varepsilon_{1}}^{+, n} \\
& +\left(D_{y}^{0} g\right)_{\wedge} \cdot \mathcal{N}_{y, \varepsilon_{1}}^{-, n}+\left(D_{y}^{0} g\right)_{\vee} \cdot \mathcal{N}_{y, \varepsilon_{1}}^{+, n} \\
& +\lambda \frac{u^{n}-f}{\sqrt{\left(u^{n}-f\right)^{2}+\varepsilon_{2}}},
\end{aligned}
$$

where $D_{x}^{0} f=\left(f_{i_{x}+1, i_{y}}-f_{i_{x}-1, i_{y}}\right) / 2, D_{x}^{+} u=u_{i_{x}+1, i_{y}}-$ $u_{i_{x}, i_{y}}$ and $D_{x}^{-} u=u_{i_{x}, i_{y}}-u_{i_{x}-1, i_{y}}$ are respectively the central, forward and backward approximations of the spatial derivatives in the $x$-direction, $\mathcal{N}_{x, \varepsilon}^{ \pm, n}=\frac{D_{x}^{ \pm} u^{n}}{\sqrt{\left(D_{x}^{ \pm} u^{n}\right)^{2}+\left(D_{y}^{ \pm} u^{n}\right)^{2}+\varepsilon}}$ is the approximation of the normal to the level sets of $u$ in the $x$-direction, the same approximations being held in the $y$-direction, $(\cdot)_{\wedge}:=\max (\cdot, 0),(\cdot)_{\vee}:=\min (\cdot, 0), \delta t$ being the temporal step, and $\varepsilon_{1}, \varepsilon_{2}$ small positive constants. In all our experiments, we choose $\delta t=5 \times 10^{-5}, \varepsilon_{1}=10^{-12}$ and $\varepsilon_{2}=10^{-4}$.

The numerical scheme defined in (8), determined from the classical Euler-Lagrange equations method, is actually a very slow segmentation method because of the regularization process of the TV-norm. Indeed, Energy $E_{1}$ is not directly minimized but the regularized version $\int g(x) \sqrt{|\nabla u|^{2}+\varepsilon_{1}}+\lambda \int \sqrt{(u-f)^{2}+\varepsilon_{2}}$ where $\varepsilon_{1}, \varepsilon_{2}$ are very small parameters to be faithful to the original energy and useful to avoid numerical instabilities. The direct consequence of this regularization parameter is the obligation to use a small temporal step to ensure a correct minimization process. Thus a large number of iterations to reach the steady state minimization solution is necessary. In other words, although it is correct, the segmentation process remains slow. For instance, let us come back to the first image, Fig. 1. This time, we consider a more challenging initial active contour because we choose a characteristic function of a small disk outside the two objects, see Fig. 3a. The two objects are now successfully segmented as we can see in Fig. $3 \mathrm{~b}$ thanks to the global minimization property of our model but the segmentation process takes 5 minutes. In the next section, we introduce a new numerical model based on a dual formulation of the TV-norm which gives a fast segmentation algorithm.

2.3 Fast Minimization Based on a Dual Formulation of the TV-Norm

Based on [17-20] and more precisely on [21], we use a convex regularization of the variational model:

$\min _{u}\left\{E_{1}(u, \lambda)=\int_{\Omega} g(x)|\nabla u| d x+\lambda \int_{\Omega}|u-f| d x\right\}$,

as follows:

$$
\begin{gathered}
\min _{u, v}\{E_{1}^{r}(u, v, \lambda, \theta)=\underbrace{\int_{\Omega} g(x)|\nabla u| d x}_{=T V_{g}(u)} \\
+\frac{1}{2 \theta} \underbrace{\int_{\Omega}(u+v-f)^{2} d x}_{\|u+v-f\|_{L^{2}}^{2}}+\lambda \underbrace{\int_{\Omega}|v| d x}_{\|v\|_{L^{1}}}\}
\end{gathered}
$$




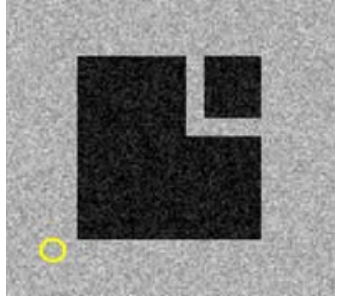

(a) Initial Active Contour.

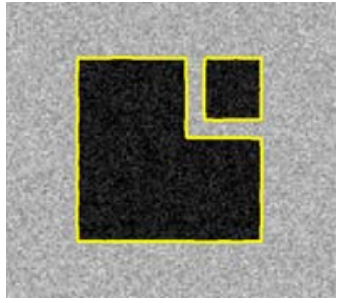

(b) Final Active Contour.

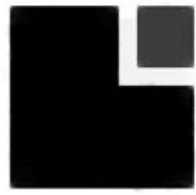

(d) Final $u$. (c) Initial $u$.

Fig. 3 Despite having an initial contour outside the two objects, a, our segmentation-denoising model successfully extracts the two meaningful objects, $\mathbf{b}$, in the given noisy image. On $\mathbf{a}, \mathbf{b}$, the active contour is given by the boundary of the set $\Omega_{C}(\mu=0.5)=\{x: u(x)>0.5\}$ and the function $u$ on $\mathbf{d}$ is the minimizer of Energy $E_{1}$, computed with the discretized flow (8). The parameter $\mu$ is arbitrary chosen to 0.5 , although any value between 0 and 1 can be used without changing the segmentation result because the final function $u$ is very close to a binary function. Hence, our snake model, based on a global minimization approach, is independent of the initial condition. This improves the standard active contour result obtained in Fig. 1 where a good initial guess is necessary to get the same result

and the parameter $\theta>0$ is small so that we almost have $f=u+v$ where the function $u$ represents the geometric information, i.e. the piecewise-smooth regions, and the function $v$ captures the texture information lying in the given image.

Since the functional $E_{1}^{r}$ is convex, its minimizer can be computed by minimizing $E_{1}^{r}$ w.r.t. $u$ and $v$ separately, and iterating until convergence as in the references mentioned above. Thus, the following minimization problems are considered:

(1) $v$ being fixed, we search for $u$ as a solution of:

$$
\min _{u}\left\{T V_{g}(u)+\frac{1}{2 \theta}\|u+v-f\|_{L^{2}}^{2}\right\} .
$$

(2) $u$ being fixed, we search for $v$ as a solution of:

$$
\min _{v}\left\{\frac{1}{2 \theta}\|u+v-f\|_{L^{2}}^{2}+\lambda\|v\|_{L^{1}}\right\} .
$$

Proposition 1 The solution of (10) is given by:

$u=f-v-\theta \operatorname{div} p$,

where $p=\left(p^{1}, p^{2}\right)$ is given by

$g(x) \nabla(\theta \operatorname{div} p-(f-v))-|\nabla(\theta \operatorname{div} p-(f-v))| p=0$.
The previous equation can be solved by a fixed point method: $p^{0}=0$ and

$p^{n+1}=\frac{p^{n}+\delta t \nabla\left(\operatorname{div} p^{n}-(f-v) / \theta\right)}{1+\frac{\delta t}{g(x)}\left|\nabla\left(\operatorname{div} p^{n}-(f-v) / \theta\right)\right|}$.

Proof See Appendix.

Proposition 2 The solution of (11) is given by:

$v= \begin{cases}f-u-\theta \lambda & \text { if } f-u \geq \theta \lambda \\ f-u+\theta \lambda & \text { if } f-u \leq-\theta \lambda \\ 0 & \text { if }|f-u| \leq \theta \lambda\end{cases}$

Proof See Appendix.

The iteration scheme (12) is straightforward to implement. The discrete divergence operator div is given by [19]:

$$
\begin{aligned}
(\operatorname{div} p)_{i_{x}, i_{y}}= & \begin{cases}p_{i_{x}, i_{y}}^{1}-p_{i_{x}-1, i_{y}}^{1} & \text { if } 1<i_{x}<N_{x}, \\
p_{i_{x}, i_{y}}^{1} & \text { if } i_{x}=1, \\
-p_{i_{x}-1, i_{y}}^{1} & \text { if } i_{x}=N_{x},\end{cases} \\
& + \begin{cases}p_{i_{x}, i_{y}}^{2}-p_{i_{x}, i_{y}-1}^{2} & \text { if } 1<i_{y}<N_{y}, \\
p_{i_{x}, i_{y}}^{2} & \text { if } i_{y}=1, \\
-p_{i_{x}, i_{y}-1}^{2} & \text { if } i_{y}=N_{y},\end{cases}
\end{aligned}
$$

and the discrete gradient operator is as follows [19]:

$(\nabla u)_{i_{x}, i_{y}}=\left((\nabla u)_{i_{x}, i_{y}}^{1},(\nabla u)_{i_{x}, i_{y}}^{2}\right)$

with

$(\nabla u)_{i_{x}, i_{y}}^{1}= \begin{cases}u_{i_{x}+1, i_{y}}-u_{i_{x}, i_{y}} & \text { if } i_{x}<N_{x}, \\ 0 & \text { if } i_{x}=N_{x},\end{cases}$
$(\nabla u)_{i_{x}, i_{y}}^{2}= \begin{cases}u_{i_{x}, i_{y}+1}-u_{i_{x}, i_{y}} & \text { if } i_{y}<N_{y}, \\ 0 & \text { if } i_{y}=N_{y} .\end{cases}$

Finally, in all experiments, initial values are chosen to be $u_{0}=v_{0}=p_{0}^{1}=p_{0}^{2}=0$, the temporal step is equal to $\delta t=$ $1 / 8$ and a stopping test is $\max \left(\left|u_{n+1}-u_{n}\right|,\left|v_{n+1}-v_{n}\right|\right) \leq \varepsilon$.

\subsection{Results}

The new active contour model, given by the global minimization of Energy (9), is applied to Fig. 1. The numerical energy minimization based on the dual formulation of the TV energy, and not on the classical technique of EulerLagrange equations such as in Sect. 2.2, gives us the same result, see Fig. 4a, in less than 5 seconds! We remind the reader that 5 minutes was necessary in the case of the EulerLagrange equations method. Furthermore, the implementation of the minimization is straightforward. Hence, our new 


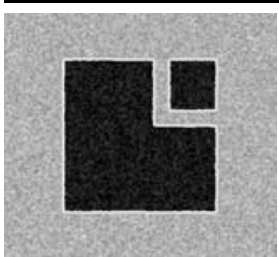

(a) Final Active Contour.

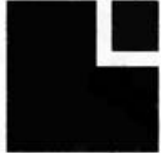

(b) Final $u$.

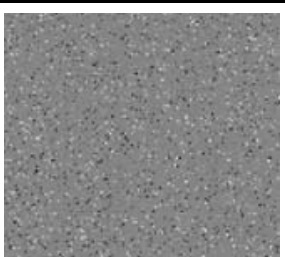

(c) Final $v$.

Fig. 4 Global minimization of the active contour/snake model with a dual formulation of the TV-norm proposed in Propositions 1 and 2. Our segmentation successfully extracts the two meaningful objects, $\mathbf{a}$, in less than 5 seconds! $\mathbf{b}$, c present the final functions $u$ and $v$ which minimize the regularized energy $E_{1}^{r}$ defined in (9). The minimization of Functional $E_{1}^{r}$ carries out the image decomposition task because $u$ represents the geometric information, i.e., the piecewise-smooth regions, and $v$ captures the texture information lying in the given image. Of course, this model also improves the standard active contour result obtained in Fig. 1 where a good initial guess is needed to get the same result. Here the initial condition is $u_{0}=v_{0}=p_{0}^{1}=p_{0}^{2}=0$. We also have $\lambda=0.1, \theta=1$

snake model provides not only a global minimum independent of the initial contour position but also an easy and fast algorithm to carry out the segmentation process. This new way to solve the active contour problem is also numerically much faster than classical methods used in [5,6] that consists of embedding the snake in a distance function and reinitializing it periodically to insure correct numerical computations of the curvature and the normal to the level sets.

It is interesting to note that our segmentation framework unifies image segmentation (snake model), image denoising (ROF model) and now image decomposition using the energy functional (9). Indeed, the introduction of the function $v$ in the minimization problem, naturally captures the textural part lying in images. Image decomposition [20, 21, 30, 31] consists of separating an image into its structural parts, representing by the geometric/piecewisesmooth regions, and textural parts, containing textures and noise. Thus the minimization of Energy (9), leading to the global minimum of the segmentation model, simultaneously performs an image decomposition which improves the segmentation task. Indeed, consider Fig. 5. The standard GAC fails to segment the rectangle, Fig. 5b, because it gets stuck in textures whereas our model, thanks to the separation between geometric regions and textures, is able to capture the black rectangle, Fig. 5c. Finally, performing image segmentation and image decomposition at the same time can be useful for other image processing tasks such as pattern recognition.

We also apply our model on a real-world image, Fig. 6(a), corrupted with a salt-and-pepper noise unlike Fig. 1 which is distorted by a Gaussian additive noise. Our model successfully extracts the meaningful part of the given image corresponding to the original text.

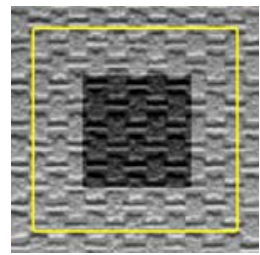

(a) Initial Standard GAC.

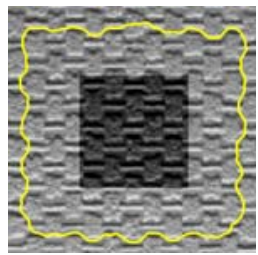

(b) Final Standard GAC.

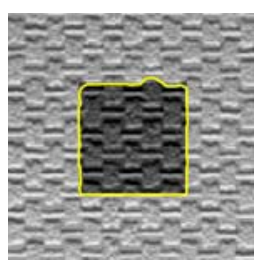

(c) "Global" Active Contour.

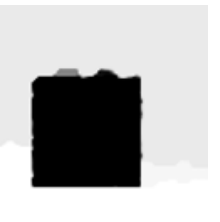

(d) Final $u$.

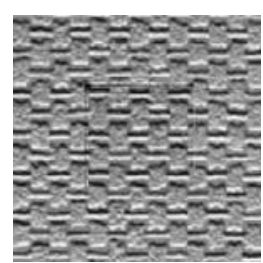

(e) Final $v$.

Fig. 5 The image decomposition used by the active contour/snake model improves the segmentation task. We mixed a black rectangle with a texture pattern. a shows the segmentation obtained with a standard GAC defined in (1-3). The standard snake fails to segment the black rectangle because it gets stuck in the textures. However, our model is able to able to capture the black rectangle, c, thanks to the image decomposition which separates the geometric part, $\mathbf{d}$, and the texture part, e, from the given image. We have $u_{0}=v_{0}=p_{0}^{1}=p_{0}^{2}=0$ and $\lambda=0.001, \theta=0.05$

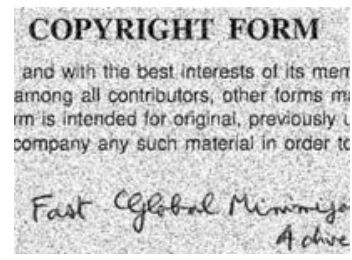

(a) Original image corrupted by a salt-and-paper noise.

\section{COPYRIGHT FORM}

and with the best interests of its men among all contributors, other forms $m$ $\mathrm{rm}$ is intended for original, previously company any such material in order tc (c) Final $u$

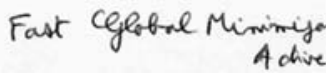

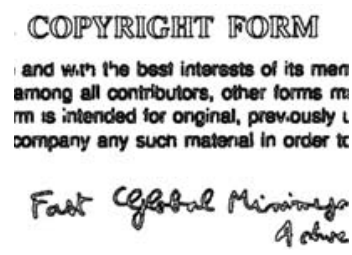

(b) Final Active Contour.

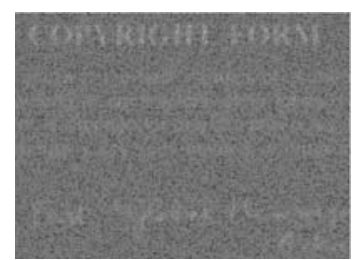

(d) Final $v$
Fig. 6 Application of our segmentation model to a real-world image. The global minimization of the snake model extracts the text, (b-c), initially corrupted by a salt-and-paper noise. The segmentation-denoising model allows us to denoise the given image and recover the original text. The advantage of the proposed snake model compared with the standard active contour model is obvious on this picture. We have $\lambda=0.0001, \theta=1$

\section{Global Minimization of the Active Contour Model Based on the Mumford-Shah Model: The Piecewise Constant Case}

The previous section defined a new image segmentation method based on the ROF model to determine a global 
minimum of the standard geodesic/geometric active contour model. This new model is thus independent of the initial contour position. However, it is designed for (noised) binary images such as Figs. 1, 5 and 6. In this section, we propose to extend the previous technique to grey-scale images.

\subsection{Theoretical Model}

We consider the global minimization problem of the active contour/snake model $[1,5,6]$ using the well-known Mumford and Shah's (MS) functional [16] and the Chan and Vese's model of active contours without edges (ACWE) [14]. The MS model is one of the most influential variational model to solve the image segmentation problem. This model determines the optimal piecewise smooth approximation of a given image, which is equivalent to partition an image into distinct homogeneous regions which boundaries are sharp and piecewise regular. The ACWE model is also an important segmentation model based on curve evolution techniques, the level set approach and the MS model. This model detects boundaries of objects based on the detection of homogeneous regions, like in the MS model, and not on the detection of large image gradients such as in the classical snake model. The efficiency of the ACWE model is presented in [14] on various experimental results for which the classical snake model, based on the image gradient, is not applicable. Chan and Vese also noticed on experimental results that their model has the tendency to compute a global minimizer. Finally, Chan, Esedoḡlu and Nikolova proved in [13] that a global minimum to the ACWE model exists.

In our approach, we propose to determine a global minimum of the snake model by enhancing the standard ACWE method. The enhancement is realized by unifying the classical GAC model with the ACWE model in a global minimization framework to detect at the same time object edges, based on the detection of large image gradients and homogeneous intensities regions. Hence, we unify the complementary approaches of the geodesic/geometric active contours model and the active contours without edges model to create an improved segmentation model. We will show that our model, besides being independent of the initial condition, improves the model of Chan and Vese when the contrast between meaningful objects and the background is low. Then, we will propose a fast numerical model, easy to implement, to carry out the image segmentation.

The variational model of ACWE, which corresponds to the two-phase piecewise constant approximation of the Mumford and Shah's model, is as follows:

$$
\begin{aligned}
& \min _{\Omega_{C}, c_{1}, c_{2}}\left\{E_{\mathrm{ACWE}}\left(\Omega_{C}, c_{1}, c_{2}, \lambda\right)=\operatorname{Per}\left(\Omega_{C}\right)\right. \\
& +\lambda \int_{\Omega_{C}}\left(c_{1}-f(x)\right)^{2} d x \\
& \left.+\lambda \int_{\Omega_{\Omega} \Omega_{C}}\left(c_{2}-f(x)\right)^{2} d x\right\},
\end{aligned}
$$

where $f$ is the given image, $\Omega_{C}$ is a closed subset of the image domain $\Omega, \operatorname{Per}\left(\Omega_{C}\right)$ is the perimeter of the set $\Omega_{C}, \lambda$ is an arbitrary positive parameter which controls the tradeoff between the regularization process and the fidelity of the solution w.r.t. the original image $f$ and $c_{1}, c_{2} \in \mathbf{R}$. The variational model (13) determines the best approximation, in the $L^{2}$ sense of the image $f$ as a set of (non-connected) regions with only two different values, $c_{1}$ and $c_{2}$. If $\Omega_{C}$ is fixed, the values of $c_{1}$ and $c_{2}$ which minimize the energy $E_{\mathrm{ACWE}}$ are the mean values inside and outside $\Omega_{C}$. Finally the term $\operatorname{Per}\left(\Omega_{C}\right)$ imposes a smoothness constraint on the geometry of the set $\Omega_{C}$ which separates the piecewise constant regions.

The minimization problem (13) is non-convex since minimization is carried over functions that take only the values $c_{1}$ and $c_{2}$, which is a non-convex collection. Hence, the optimization problem can have local minima, which implies solutions with wrong scales of details. Despite the nonconvex nature of (13), a natural way to determine a solution $\left(\Omega_{C}, c_{1}, c_{2}\right)$ is a two-step algorithm where $c_{1}$ and $c_{2}$ are first computed, then the region $\Omega_{C}$ is updated to decrease the energy $E_{\mathrm{ACWE}}$. Chan and Vese proposed in [14] a solution to determine an evolution equation for the region $\Omega_{C}$ based on a level set based approach. They represent the regions $\Omega_{C}$ and $\Omega \backslash \Omega_{C}$ with the Heaviside function of a level set function (which models a characteristic function). Hence the energy $E_{\mathrm{ACWE}}$ can be written according to a level set function $\phi$ :

$$
\begin{aligned}
& E_{\mathrm{ACWE}}^{2}\left(\phi, c_{1}, c_{2}, \lambda\right)=\int_{\Omega}\left|\nabla H_{\epsilon}(\phi)\right| d x \\
& \quad+\lambda \int_{\Omega}\left(H_{\epsilon}(\phi)\left(c_{1}-f(x)\right)^{2}+H_{\epsilon}(-\phi)\left(c_{2}-f(x)\right)^{2}\right) d x,
\end{aligned}
$$

where $\Omega$ is the image domain and $H_{\epsilon}$ is a regularization of the Heaviside function. The flow minimizing Energy (14) is the following one:

$$
\begin{aligned}
\partial_{t} \phi= & H_{\epsilon}^{\prime}(\phi)\left\{\operatorname{div}\left(\frac{\nabla \phi}{|\nabla \phi|}\right)\right. \\
& -\lambda \underbrace{\left(\left(c_{1}-f(x)\right)^{2}-\left(c_{2}-f(x)\right)^{2}\right)}_{=: r_{1}\left(x, c_{1}, c_{2}\right)}\} .
\end{aligned}
$$

In [14], authors chose a non-compactly supported smooth strictly monotone approximation of the Heaviside function. As a result, the steady state solution of the gradient flow (15) is the same as:

$\partial_{t} \phi=\operatorname{div}\left(\frac{\nabla \phi}{|\nabla \phi|}\right)-\lambda r_{1}\left(x, c_{1}, c_{2}\right)$, 
and this equation is the gradient descent flow of the following energy:

$$
\begin{aligned}
E_{\mathrm{ACWE}}^{3}\left(\phi, c_{1}, c_{2}, \lambda\right)= & \int_{\Omega}|\nabla \phi| d x \\
& +\lambda \int_{\Omega} r_{1}\left(x, c_{1}, c_{2}\right) \phi d x .
\end{aligned}
$$

Based on the previous development, we propose to minimize the following energy functional, for any given observed image $f \in L^{1}(\Omega)$ and any parameter $\lambda>0$, to carry out the global minimization of the segmentation task:

$$
E_{2}\left(u, c_{1}, c_{2}, \lambda\right):=T V_{g}(u)+\lambda \int_{\Omega} r_{1}\left(x, c_{1}, c_{2}\right) u d x .
$$

The difference between Energy (17) and (16) is based on the weighted total variation energy, $T V_{g}(u)$, of the function $u$ with a weight function $g$. This simple modification gives us the link between the ACWE model and the standard snake model when the function $g$ is an edge indicator function and the function $u$ is a characteristic function, $\mathbf{1}_{\Omega_{C}}$. Indeed, Energy (17) is in the case of characteristic functions equal to:

$$
\begin{aligned}
& E_{2}\left(u=\mathbf{1}_{\Omega_{C}}, c_{1}, c_{2}, \lambda\right) \\
& \quad=T V_{g}\left(\mathbf{1}_{\Omega_{C}}\right)+\lambda \int_{\Omega} r_{1}\left(x, c_{1}, c_{2}\right) \mathbf{1}_{\Omega_{C}} d x, \\
& \quad=\int_{C} g d s+\lambda \int_{\Omega}\left(\left(c_{1}-f(x)\right)^{2}-\left(c_{2}-f(x)\right)^{2}\right) \mathbf{1}_{\Omega_{C}} d x .
\end{aligned}
$$

Hence, minimizing Energy (18) is equivalent to

minimize $\int_{C} g d s=E_{\mathrm{GAC}}(C)$ (the snake/GAC energy (1)), while

approximating $f$ (in the $L^{2}$ sense) by two regions $\Omega_{C}$ and $\Omega \backslash \Omega_{C}$ with two values $c_{1}$ and $c_{2}$.

The previous observation, about the energy $E_{2}$ and the characteristic functions of sets, emphasizes the link between the standard active contour model $[1,5,6]$ and the ACWE model [14]. Moreover, Energy $E_{2}$ also provides us a global minimum for the active contour model. The following theorem states the existence of a global minimizer for Energy $E_{2}$ :

Theorem 2 Suppose that $f(x), g(x) \in[0,1]$, for any given $c_{1}, c_{2} \in \mathbf{R}$ and $\lambda \in \mathbf{R}_{+}$, if $u(x)$ is any minimizer of $E_{2}\left(., c_{1}\right.$, $\left.c_{2}, \lambda\right)$, then for almost every $\mu \in[0,1]$ we have that the characteristic function

$\mathbf{1}_{\Omega_{C}(\mu)=\{x: u(x)>\mu\}}(x)$,

where $C$ is the boundary of the set $\Omega_{C}$, is a global minimizer of $E_{2}\left(., c_{1}, c_{2}, \lambda\right)$.
Proof See Appendix.

The interpretation of Theorem 2 is as follows: for $\lambda, c_{1}, c_{2}$ being fixed, any minimizer $u$ of $E_{2}$, determined with any minimization technique such as the EulerLagrange equations method or another optimization method, the set of points in the function $u$ such as $u$ is larger to an arbitrary positive constant, e.g. $\mu=0.5$, defines a set $\Omega_{C}$ whose boundary $C$ represents a global minimum of the snake model subject to intensity homogeneity constraints.

Like the energy of ACWE [13], Energy $E_{2}$ is homogeneous of degree 1 in $u$. This means that this evolution equation does not have a stationary solution if the minimization to $u$ is not restricted such as $0 \leq u(x) \leq 1$. Thus, the constrained minimization problem to carry out the segmentation task is in fact as follows:

$$
\begin{aligned}
& \min _{0 \leq u \leq 1}\left\{E_{2}\left(u, c_{1}, c_{2}, \lambda\right)\right. \\
& \left.=T V_{g}(u)+\lambda \int_{\Omega} r_{1}\left(x, c_{1}, c_{2}\right) u d x\right\} .
\end{aligned}
$$

The constrained problem (19) is changed into an unconstrained minimization problem according to the following theorem [13]:

Theorem 3 Let $r_{1}\left(x, c_{1}, c_{2}\right) \in L^{\infty}(\Omega)$, for any given $c_{1}$, $c_{2} \in \mathbf{R}$ and $\lambda \in \mathbf{R}_{+}$, then the following convex constrained minimization problem

$$
\min _{0 \leq u \leq 1}\left\{T V_{g}(u)+\lambda \int_{\Omega} r_{1}\left(x, c_{1}, c_{2}\right) u d x\right\}
$$

has the same set of minimizers as the following convex and unconstrained minimization problem:

$\min _{u}\left\{T V_{g}(u)+\int_{\Omega} \lambda r_{1}\left(x, c_{1}, c_{2}\right) u+\alpha v(u) d x\right\}$

where $v(\xi):=\max \left\{0,2\left|\xi-\frac{1}{2}\right|-1\right\}$ is an exact penalty function provided that the constant $\alpha$ is chosen large enough compared to $\lambda$ such as $\alpha>\frac{\lambda}{2}\left\|r_{1}(x)\right\|_{L^{\infty}(\Omega)}$.

Proof See Appendix.

Like in Sect. 2.1, Energy $E_{3}$ given by:

$$
\begin{aligned}
E_{3}\left(u, c_{1}, c_{2}, \lambda, \alpha\right):= & T V_{g}(u) \\
& +\int_{\Omega} \lambda r_{1}\left(x, c_{1}, c_{2}\right) u+\alpha \nu(u) d x,
\end{aligned}
$$

is convex but not strictly convex, which mean that $E_{3}$ does not possess local minima that are not global minima. Hence any minimizer of Energy $E_{3}$ is a global minimizer. As we did in Sect. 2.2, we could compute a global minimizer of $E_{3}$ 
with the standard Euler-Lagrange equations technique and the explicit gradient descent based algorithm (see [32] for numerical details). However, as we explained in Sect. 2.2, this numerical minimization method is very slow because of the regularization of the TV-norm. Thus, we introduce in the next section a new numerical model, based on a dual formulation of the TV-norm, which will define a fast segmentation algorithm, much faster than the standard snake model.

3.2 Fast Minimization Based on a Dual Formulation of the TV-norm

The variational problem:

$$
\begin{aligned}
& \min _{u}\left\{E_{3}\left(u, c_{1}, c_{2}, \lambda, \alpha\right)\right. \\
& \left.\quad=T V_{g}(u)+\int_{\Omega} \lambda r_{1}\left(x, c_{1}, c_{2}\right) u+\alpha v(u) d x\right\}
\end{aligned}
$$

is regularized in the same way as in Sect. 2.3 based on [17-21]:

$$
\begin{aligned}
\min _{u, v}\left\{E_{3}^{r}\left(u, v, c_{1}, c_{2}, \lambda, \alpha, \theta\right)\right. \\
=T V_{g}(u)+\frac{1}{2 \theta}\|u-v\|_{L^{2}}^{2} \\
\left.\quad+\int_{\Omega} \lambda r_{1}\left(x, c_{1}, c_{2}\right) v+\alpha v(v) d x\right\},
\end{aligned}
$$

where the parameter $\theta>0$ is chosen to be small. Since Functional $E_{3}^{r}$ is convex, its minimizer can be computed by minimizing $E_{3}^{r}$ w.r.t. $u$ and $v$ separately, and to iterate until convergence. Thus, the following minimization problems are considered:

(1) $v$ being fixed, we search for $u$ as a solution of:

$$
\min _{u}\left\{T V_{g}(u)+\frac{1}{2 \theta}\|u-v\|_{L^{2}}^{2}\right\} .
$$

(2) $u$ being fixed, we search for $v$ as a solution of:

$$
\begin{aligned}
\min _{v}\left\{\frac{1}{2 \theta}\|u-v\|_{L^{2}}^{2}+\int_{\Omega} \lambda r_{1}\left(x, c_{1}, c_{2}\right) v\right. \\
+\alpha v(v) d x\} .
\end{aligned}
$$

Proposition 3 The solution of (21) is given by:

$u=v-\theta \operatorname{div} p$,

where $p=\left(p^{1}, p^{2}\right)$ is given by

$g(x) \nabla(\theta \operatorname{div} p-v)-|\nabla(\theta \operatorname{div} p-v)| p=0$.
The previous equation can be solved by a fixed point method: $p^{0}=0$ and

$p^{n+1}=\frac{p^{n}+\delta t \nabla\left(\operatorname{div}\left(p^{n}\right)-v / \theta\right)}{1+\frac{\delta t}{g(x)}\left|\nabla\left(\operatorname{div}\left(p^{n}\right)-v / \theta\right)\right|}$.

Proof The proof is the same as Proposition 1 when $f-v$ is replaced by $v$.

Proposition 4 The solution of (22) is given by:

$v=\min \left\{\max \left\{u(x)-\theta \lambda r_{1}\left(x, c_{1}, c_{2}\right), 0\right\}, 1\right\}$.

Proof See Appendix.

The iteration scheme (23) is straightforward to implement as in Sect. 2.3. Thus (23) and (24) are iterated in order to minimize Energy (20). Of course, the constants $c_{1}$ and $c_{2}$ are updated periodically every 10 iterations. In all experiments, initial values are $u_{0}=v_{0}=p_{0}^{1}=p_{0}^{2}=0$, the temporal step is equal to $\delta t=1 / 8$ and the stopping criteria is $\max \left(\left|u_{n+1}-u_{n}\right|,\left|v_{n+1}-v_{n}\right|\right) \leq \varepsilon$.

\subsection{Results}

The new snake model, given by the global minimization of Energy (20), is applied to the cameraman picture, Fig. 7. The numerical minimization based on the dual formulation of the TV-norm, and not on the classical technique of the EulerLagrange equations, gives us the same result, Fig. 7a, in less than 10 seconds! Furthermore, as we noticed in Sect. 2.4, the implementation of the minimization is straightforward, fast

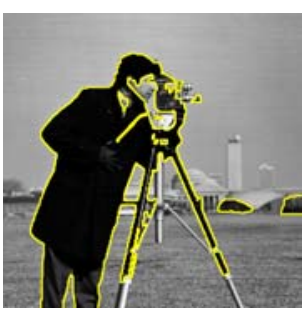

(a) Final Active Contour.

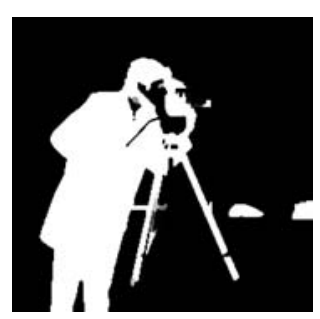

(b) Final $u$.
Fig. 7 Global minimization of the active contour/snake model using the Mumford-Shah's model and the Chan-Vese's model. Our segmentation model "reconciles" in a consistent framework the standard GAC model, based on the detection of edge points defined by large image gradients, and the ACWE model, based on the detection of homogeneous regions defined from the Mumford-Shah energy. A minimization of $E_{2}$ realized with the Euler-Lagrange equations technique takes about 10 minutes. Here, the numerical minimization of $E_{2}$, given in $\mathbf{b}$, is carried out with a dual formulation of the TV-norm in less than 10 seconds! As in Sect. 2, the active contour in a is given by the boundary of the set $\Omega_{C}(\mu=0.5)=\{x: u(x)>0.5\}$. The parameter $\mu$ is arbitrary chosen to 0.5 , even if any value between 0 and 1 can be used without changing the segmentation result because the final function $u$ is very close to a binary function. We choose $\lambda=0.1, \theta=1$ 


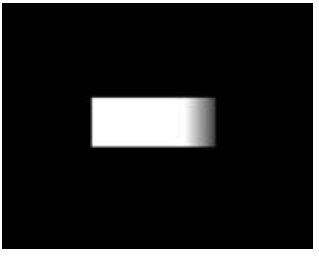

(a) Original image.

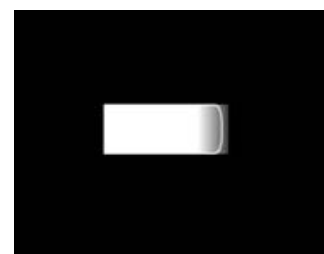

(b) Final Active Contour given by the ACWE model.

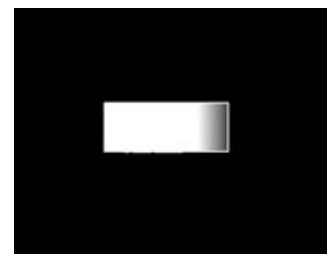

(c) Final Active Contour given by our model.
Fig. 8 Segmentation using the Active Contours Without Edges (ACWE) model of Chan-Vese, $\mathbf{b}$, and our model which unifies the ACWE and the geodesic/geometric active contours (GAC), c. This synthetic image illustrates one important advantage of our segmentation model over the standard ACWE model. Indeed, whatever the value of the fidelity constant $\lambda$, the ACWE model can not fully segment the rectangular foreground object. It will always prefer to cut through the low contrast region of the foreground object, see $\mathbf{b}$. However, our segmentation algorithm is able to fully capture the boundary of the foreground object, c, even though the contrast changes are very low at the right edge of the rectangular object, because the model uses the edge indicator function $g$ defined in the GAC model. We choose $\lambda=0.01, \theta=1$ for $\mathbf{b}$ and $\lambda=0.0001, \theta=1$ for $\mathbf{c}$

and independent of the initial condition (we simply chose $u_{0}=v_{0}=p_{0}^{1}=p_{0}^{2}=0$ in Fig. 7). Hence, this new way to solve the active contour propagation problem is numerically more efficient than classical methods, which consists of embedding the snake in a distance function and re-initializing it periodically to insure correct computations of the curvature and the normal to the level sets.

Our segmentation model improves not only the GAC model but also the ACWE model when the contrast between meaningful objects and the background is low. Indeed, let us consider the synthetic image in Fig. 8a. At the right edge of the rectangular foreground object, the contrast changes are very low even though there is still a clear discontinuity delineating the edge. The result obtained using the standard ACWE model is shown in Fig. 8b. No matter how large the fidelity constant $\lambda$ is chosen, the model will always prefer to cut through the low contrast region of the foreground object (does so exactly where the contrast is 0.5 ). There is no way to avoid this by varying the parameters in the model, the active contour always misses the correct boundary at the right edge of the rectangle by at least the amount shown. However, the solution obtained using our segmentation algorithm, shown in Fig. 8c, provides enough of edge sensitivity, given by the edge indicator function $g$, for the active contour to stay faithful to the actual boundary of the foreground object.

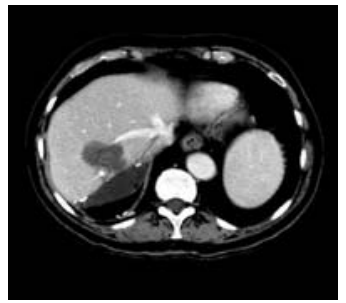

(a) Original image.

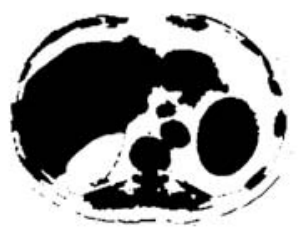

(c) Final $u$.

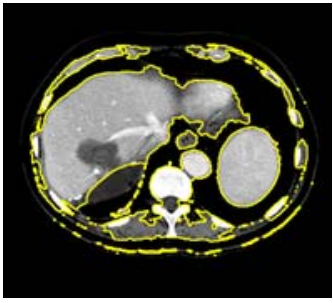

(b) Final Active Contour.

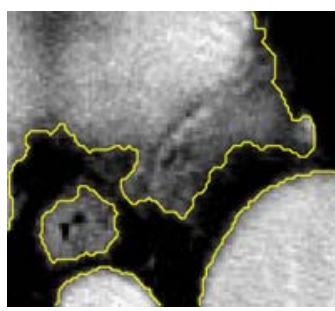

(d) Final Active Contour.
Fig. 9 Our segmentation model is able to segment an important part of the liver despite of very low contrast changes, b-d. The fine segmentation result is obtained by unifying the ACWE model and the GAC model which accurately detects boundaries thanks to the edge indicator function $g$. The Standard ACWE can not segment as accurately as our model as shown in Fig. 10. We choose $\lambda=0.5, \theta=0.1$

We show the advantage of our model over the standard ACWE model on a real-world image, Fig. 9a. Our model is able to segment an important part of the liver, Figs. $9 \mathrm{~b}$ and d, despite of very low contrast changes, whereas the standard ACWE model can not segment accurately the liver as we can see in Fig. 10 where different values of $\lambda$ was tested.

\section{Global Minimization of the Active Contour Model Based on the Mumford-Shah Model: The Piecewise Smooth Case}

The previous section defined an image segmentation model based on the two-phase piecewise constant approximation, also known as the cartoon version, of the MS model to determine a minimum of the snake model independently of the initial contour position. In the following section, we extend the previous model to the two-phase piecewise smooth approximation of the MS model. The variational problem to solve is given by Vese and Chan (VC) in [33] (see also [34] for early work) by:

$$
\begin{aligned}
& \min _{\Omega_{C}, s_{1}, s_{2}}\left\{E_{V C}\left(\Omega_{C}, s_{1}, s_{2}, \eta, \lambda\right)=\operatorname{Per}\left(\Omega_{C}\right)\right. \\
& \quad+\lambda \int_{\Omega_{C}}\left(\left(s_{1}(x)-f(x)\right)^{2}+\eta\left|\nabla s_{1}(x)\right|^{2}\right) d x \\
& \left.\quad+\lambda \int_{\Omega_{\backslash} \Omega_{C}}\left(\left(s_{2}(x)-f(x)\right)^{2}+\eta\left|\nabla s_{2}(x)\right|^{2}\right) d x\right\},
\end{aligned}
$$




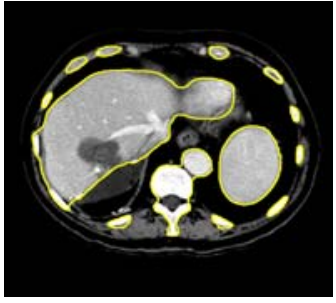

(a) Final ACWE with $\lambda=$ 0.0001 .

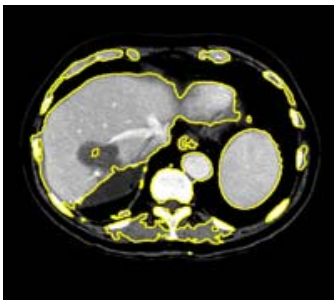

(c) Final ACWE with $\lambda=$ 0.001 .

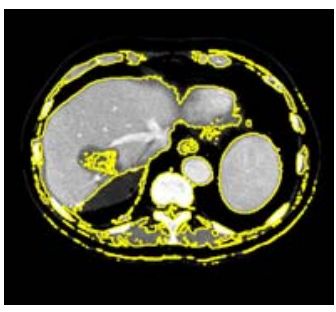

(e) Final ACWE with $\lambda=$ 0.01 .

Fig. 10 Segmentation using the ACWE model. Whatever the fidelity constant $\lambda$, the ACWE can not produce the same result obtained in Figs. $9 \mathrm{~b}$ and $\mathrm{d}$ because it does not use an edge indicator function $g . \mathbf{a}-\mathbf{f}$ presents different results given by different values of $\lambda$

where $\Omega$ is the image domain, $f$ is the given image, $s_{1}$ and $s_{2}$ are two $C^{1}$ functions defined on $\Omega_{C}$ and on $\Omega \backslash \Omega_{C}$ respectively, $\lambda>0$ controls the regularization of the length of the boundary of smooth regions and $\eta>0$ controls the regularization of the intensities of smooth regions. The variational problem (25) determines the best approximation, in the $L^{2}$ sense, of the given image $f$ as a set of smooth regions represented by the function $s(x)$ such that

$s(x):= \begin{cases}s_{1}(x) & \text { if } x \in \Omega_{C}, \\ s_{2}(x) & \text { if } x \in \Omega \backslash \Omega_{C},\end{cases}$

and $C=\partial \Omega_{C}=\partial\left(\Omega \backslash \Omega_{C}\right)$ is the boundary between the smooth regions. Like (13), the minimization problem (25) is also non-convex, which implies the existence of local minima and possibly unsatisfactory segmentation results. As in Sect. 3.1, the two regions $\Omega_{C}$ and $\Omega \backslash \Omega_{C}$ are represented by a regularized Heaviside function, $H_{\epsilon}($.$) , of a level set func-$ tion $\phi$. This leads to the following energy:

$$
\begin{aligned}
& E_{C V}^{2}\left(\Omega_{C}, s_{1}, s_{2}, \eta, \lambda\right) \\
& =\int_{\Omega}\left|\nabla H_{\epsilon}(\phi)\right| d x+\lambda \int_{\Omega} H_{\epsilon}(\phi)\left(\left(s_{1}-f\right)^{2}+\eta\left|\nabla s_{1}\right|^{2}\right) d x \\
& \quad+\lambda \int_{\Omega} H_{\epsilon}(-\phi)\left(\left(s_{2}-f\right)^{2} \eta\left|\nabla s_{2}\right|^{2}\right) d x .
\end{aligned}
$$

Minimizing $E_{C V}^{2}$ w.r.t. the functions $s_{1}$ and $s_{2}$ using the calculus of variations gives us:

$\begin{cases}s_{1}-f=\eta \Delta s_{1} & \text { in } \Omega_{C}, \\ s_{2}-f=\eta \Delta s_{2} & \text { in } \Omega \backslash \Omega_{C},\end{cases}$

with the Neumann boundary conditions:

$\begin{cases}\frac{\partial s_{1}}{\partial \mathcal{N}}=0 & \text { on } \partial \Omega_{C} \cup \partial \Omega, \\ \frac{\partial s_{2}}{\partial \mathcal{N}}=0 & \text { on } \partial\left(\Omega \backslash \Omega_{C}\right) \cup \partial \Omega .\end{cases}$

And the flow minimizing the energy (26) is as follows:

$$
\begin{aligned}
& \partial_{t} \phi=H_{\epsilon}^{\prime}(\phi)\left\{\operatorname{div}\left(\frac{\nabla \phi}{|\nabla \phi|}\right)\right. \\
&-\lambda \underbrace{\left(\left(s_{1}-f\right)^{2}-\left(s_{2}-f\right)^{2}+\eta\left|\nabla s_{1}\right|^{2}-\eta\left|\nabla s_{2}\right|^{2}\right)}_{=: r_{2}\left(x, s_{1}, s_{2}, \eta\right)}\} .
\end{aligned}
$$

If a non-compactly supported smooth approximation of the Heaviside function is chosen, the steady state solution of the gradient flow (28) is the same as:

$\partial_{t} \phi=\operatorname{div}\left(\frac{\nabla \phi}{|\nabla \phi|}\right)-\lambda r_{2}\left(x, s_{1}, s_{2}, \eta\right)$

and this equation is the gradient descent flow of the energy:

$$
\begin{aligned}
& E_{C V}^{3}\left(\phi, s_{1}, s_{2}, \eta, \lambda\right) \\
& \quad=\int_{\Omega}|\nabla \phi|+\lambda \int_{\Omega} r_{2}\left(x, s_{1}, s_{2}, \eta\right) \phi d x .
\end{aligned}
$$

As a result, the following constrained minimization problem is proposed for any given image $f \in L^{1}(\Omega)$ and any positive parameter $\lambda>0$ :

$$
\begin{aligned}
& \min _{0 \leq u \leq 1}\left\{E_{3}\left(u, s_{1}, s_{2}, \eta, \lambda\right)\right. \\
& \left.=\int_{\Omega} g|\nabla u| d x+\lambda \int_{\Omega} r_{2}\left(x, s_{1}, s_{2}, \eta\right) u d x\right\} .
\end{aligned}
$$

We point out that the two functions $s_{1}$ and $s_{2}$ in the formulation (26) need to be defined only on their respective domains (namely $\Omega_{C}$ and $\Omega \backslash \Omega_{C}$ ) because of the Heaviside function. However, in the relaxed formulation given in (30), these functions need to be defined in the entire domain $\Omega$ (by a suitable extension). 
The difference between Energy (29) and (30) is based on the weighted total variation energy, $T V_{g}(u)$, which gives us the link between the GAC model and the MS model when the function $g$ is an edge indicator function and the function $u$ is a characteristic function, $\mathbf{1}_{\Omega_{C}}$. Indeed, Energy (30) in the case of characteristic functions is equal to:

$$
\begin{aligned}
& E_{3}\left(u=\mathbf{1}_{\Omega_{C}}, s_{1}, s_{2}, \eta, \lambda\right) \\
& =\int_{\Omega} g\left|\nabla \mathbf{1}_{\Omega_{C}}\right| d x+\lambda \int_{\Omega} r_{2}(x) \mathbf{1}_{\Omega_{C}} d x, \\
& =\int_{C} g d s+\lambda \int_{\Omega}\left(\left(s_{1}-f\right)^{2}-\left(s_{2}-f\right)^{2}\right. \\
& \left.\quad+\eta\left|\nabla s_{1}\right|^{2}-\eta\left|\nabla s_{2}\right|^{2}\right) \mathbf{1}_{\Omega_{C}} d x .
\end{aligned}
$$

Hence, minimizing Energy (31) is equivalent to

minimize $\int_{C} g d s=E_{\mathrm{GAC}}(C)$ (the snake/GAC energy (1)), while

approximating $f$ (in the $L^{2}$ sense) by two piecewise smooth regions $\Omega_{C}$ and $\Omega \backslash \Omega_{C}$.

The previous observation, about Energy $E_{3}$ and characteristic functions of sets, emphasizes the relation between the standard active contour model $[1,5,6]$ and the $\mathrm{VC}$ model [33]. Besides, Energy $E_{3}$ also provides us a global minimum for the active contour model. Indeed, the following theorem states the existence of a global minimizer for Energy $E_{3}$ :

Theorem 4 Suppose that $f(x), g(x) \in[0,1]$, for any given $s_{1}, s_{2} \in C^{1}(\Omega)$ and $\lambda, \eta \in \mathbf{R}_{+}$, if $u(x)$ is any minimizer of $E_{3}\left(., s_{1}, s_{2}, \eta, \lambda\right)$, then for almost every $\mu \in[0,1]$ we have that the characteristic function

$\mathbf{1}_{\Omega_{C}(\mu)=\{x: u(x)>\mu\}}(x)$,

where $C$ is the boundary of the set $\Omega_{C}$, is a global minimizer of $E_{3}\left(., s_{1}, s_{2}, \eta, \lambda\right)$.

Proof The proof is similar to the proofs of Theorems 2 and 3 when the function $r_{1}$ is replaced by the function $r_{2}$.

The interpretation of Theorem 4 is as follows: for $s_{1}, s_{2}, \eta, \lambda$ being fixed, any minimizer $u$ of $E_{3}$, determined with any minimization technique such as the EulerLagrange equations method or another optimization method, the set of points in the function $u$ such as $u$ is larger to an arbitrary positive constant, e.g., $\mu=0.5$, defines a set $\Omega_{C}$ whose boundary $C$ represents a global minimum of the snake model.

Finally, a minimizer of the energy $E_{3}$ can be found using the Euler-Lagrange equations technique like in Sect. 2.2

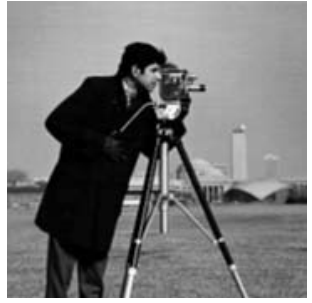

(a) Original Image.

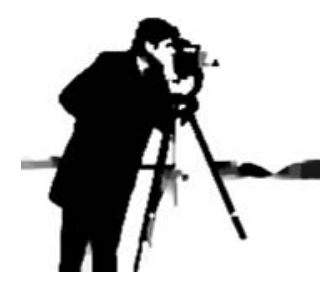

(c) Final $u$.

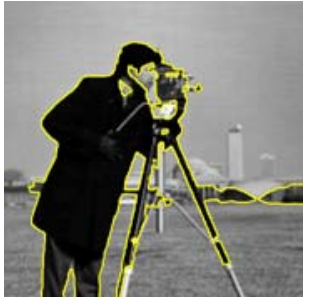

(b) Final Active Contour.

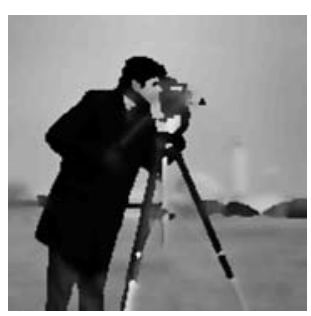

(d) Smooth approximation of (a).
Fig. 11 Segmentation using the GAC model and the two-phase piecewise smooth approximation of the MS model defined by Vese-Chan in [33]. $\mathbf{b}$ presents the final active contour and $\mathbf{d}$ the optimal two-phase piecewise smooth approximation of the given image

(see [32] for numerical details) or the dual formulation of the TV-norm like in Sect. 3.2. Figure 11b presents the segmentation of the cameraman picture carrying out by the minimization of energy $E_{3}$ and Theorem 4. Figure 11d shows the optimal two-phase piecewise smooth approximation of the original image given by the MS model. Notice that the two functions $s_{1}, s_{2}$ are initially chosen to $f$ and updated every 10 iterations according to (27). Finally, Fig. 12 present the segmentation and the denoising of a smooth foreground object.

\section{Comparison with Related Works}

In this section, we consider three works related with our approach.

The first related approach is naturally the work of Chan, Esedoğlu and Nikolova [13] because the global minimization of the snake/GAC model proposed in this paper is inspired by their work. However, our approach is more general because we propose a unified framework to use the GAC model and the ACWE model. Experimental results in Sect. 3.3 demonstrate the advantage of using the GAC model with the ACWE model when the contrast changes between meaningful objects and the background are very low. Finally, we propose a fast numerical scheme to perform the global minimization of our variational model, which is not the case in [13].

The second related work is the paper [35] of Cohen and Kimmel which also addresses the problem of determining 


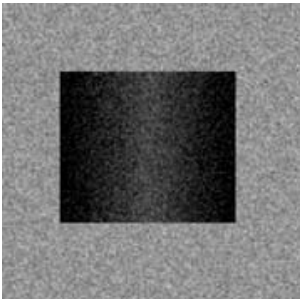

(a) Original Image.

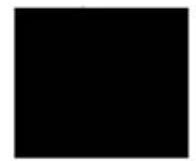

(c) Initial $u$

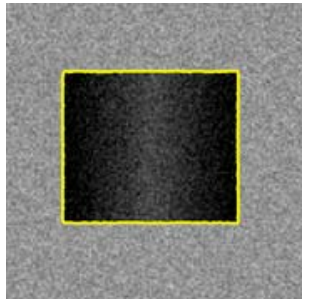

(b) Final Active Contour.

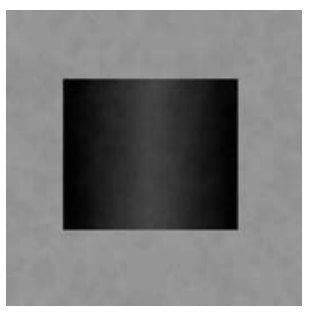

(d) Denoised Image.
Fig. 12 Segmentation using the GAC model and the two-phase piecewise smooth approximation of the MS model defined by Vese-Chan in [33]. Our segmentation model also performs at the same time the image denoising because $\mathbf{d}$ presents the regularized version of $\mathbf{a}$

a global minimum for the GAC energy. Their approach is different from ours since it is focused on finding a minimal path between two given end points of an open curve. As noticed in [36], object segmentation is not easy to carry out in their approach because the method needs a number of points on the boundary of the object to be extracted. Furthermore the model is naturally designed to capture open curves, such as minimal paths on road images, but not directly closed curves because it requires a complementary method based on a topology-based saddle search routine.

Finally, in [36], Appleton and Talbot propose to determine a global minimum for the GAC model for closed curves under the restriction that the curve contains a specified internal point. Authors present very good object segmentation results in various medical images. However, the need of a specified internal point can limit the segmentation process because it means that object with multiple closed curves can not be extracted without a set of seed points. For example, the two objects presented in Fig. 1 can not be directly segmented with only one internal point. Their model needs to detect two internal points, which is not our case. Finally, the extension of their model to higher-dimensional images is not straightforward whereas the extension is natural in our approach.

\section{Conclusion}

As we said at the beginning, the active contour/snake model is a well-known image segmentation model which is more and more used in various image processing applications such as in automated surveillance, graphics animation, robotics or medical imaging. Its success is based on strong theoretical properties and efficient numerical schemes. The only drawback of this segmentation model is the existence of local minima in its functional energy, which makes critical the initial contour to extract meaningful objects lying in images. Hence we proposed in this paper a new approach to determine a global minimum of the snake energy in order to become independent of the initial position of the contour. We think that this new approach can have numerous applications in the image processing tasks previously mentioned.

The core of our models was to express the energy functionals in terms of level sets as observed by Strang in $[37,38]$ and solve geometric problems as proposed by Chan-Esedoḡlu-Nikolova [13]. Thus we defined three new variational models based on the unification of the classical snake/GAC model [1, 5, 6], the denoising Rudin-OsherFatemi model [15], the segmentation Mumford-Shah model [16] and the active contours without edges model [14]. In the case of the ROF model, we obtained a global minimization theorem for binary images. It was interesting to notice that the computation of the global minimum was done by decomposing an image into a geometric part, i.e., smooth part, and a texture part as in [20, 21]. Experimental results showed that the image decomposition improves the segmentation task. In the case of the ACWE model of Chan-Vese for grey-scale images, we showed that our model improves classical segmentation results at the location of smooth transitions between objects and the background thanks to the edge indicator function.

We established theorems to prove the existence of global minimizers to our segmentation variational models. We determined not one but several global minima of the active contour model, which looks to be a drawback. However, all global solutions are close to each other because the minimizers $u$ are very close to binary functions.

We also proposed efficient and fast numerical schemes to globally minimize the variational segmentation models. The proposed algorithms, based on a dual formulation of the TVnorm proposed and developed in [17-21], are easy to implement. This new way to solve the standard contour propagation problem allows us to avoid the usual drawback in the level set approach that consists of initializing the active contour in a distance function and re-initializing it periodically during the evolution to ensure a correct computation of the curvature and the normal to the level sets, which is timeconsuming. See [39] for more details and another approach to solve the re-distancing problem.

Future works will investigate the extension of this global minimization approach to other image processing variational models, which most of them suffers from the existence of local minima. One application of our work has been done in [40] to unify image segmentation, image denoising and image inpainting. 
Acknowledgements The authors would like to thank the unknown reviewers for their general and detailed comments.

\section{Appendix}

Definition 1 Let $\Omega \subset \mathbf{R}^{N}$ be an open set, $u \in L^{1}(\Omega)$ and $g$ a positive valued continuous and bounded function in $\Omega$. The weighted total variation norm of $u$ with the weight function $g$ is defined by

$T V_{g}(u)=\int_{\Omega} g(x)|\nabla u| d x=\sup _{\varphi \in \Phi_{g}}\left\{\int_{\Omega} u(x) \operatorname{div} \varphi(x) d x\right\}$,

where

$\Phi_{g}:=\left\{\varphi \in C^{1}(\Omega, \mathbf{R}):|\varphi(x)| \leq g\right.$, for all $\left.x \in \Omega\right\}$.

Strang in [38] defines the coarea formula for the $T V_{g}$ norm as follows:

$$
\begin{aligned}
\int_{\Omega} g|\nabla u| & =\int_{-\infty}^{\infty}\left(\int_{\gamma_{\mu}} g d s\right) d \mu \\
& =\int_{-\infty}^{\infty} \operatorname{Per}_{g}\left(E_{\mu}:=\{x: u(x)>\mu\}\right) d \mu,
\end{aligned}
$$

where $\gamma_{\mu}$ is the boundary of the set $E_{\mu}$ on which $u(x)>\mu$. Hence, the term $\operatorname{Per}_{g}\left(E_{\mu}\right)=\int_{\gamma_{\mu}} g d s$ is the perimeter of the set $E_{\mu}$ weighted by the function $g$.

Proof of Theorem 1 The proof is in [13], based on [37, 38], by replacing the TV-norm by the weighted TV-norm introduced in Definition 1. It basically consists of expressing Energy $E_{1}$, defined in (5), in terms of the level sets of $u$ and $f$ and solving a geometric problem point-wise in $\mu$.

Proof of Proposition 1 The proof is based on [17, 19]. The $u$-minimization in $E_{1}^{r}$ is

$\min _{u}\left\{\int_{\Omega} g(x)|\nabla u| d x+\frac{1}{2 \theta} \int_{\Omega}(u-\underbrace{(f-v)}_{=: f_{2}})^{2} d x\right\}$.

We proceed exactly as in [17] and [19]. As shown in [17], (32) can be written with the dual variable $p=\left(p_{1}, p_{2}\right)$ :

$\min _{u} \max _{|p| \leq g} \int_{\Omega} u \operatorname{div} p+\frac{1}{2 \theta}\left(u-f_{2}\right)^{2} d x$.

One can now switch the min and the max to obtain the equivalent

$\max _{|p| \leq g} \min _{u} \int_{\Omega} u \operatorname{div} p+\frac{1}{2 \theta}\left(u-f_{2}\right)^{2} d x$.
The inner minimization in (33) is point-wise in $u$. Carrying it out gives:

$\operatorname{div} p+\frac{1}{\theta}\left(u-f_{2}\right)=0 \Rightarrow u=f-v-\theta \operatorname{div} p$.

Substituting the expression (34) for minimal $u$ into the maxmin problem (33) gives

$\max _{|p| \leq g} \int_{\Omega}\left(f_{2}-\theta \operatorname{div} p\right) \operatorname{div} p+\frac{\theta}{2}(\operatorname{div} p)^{2} d x$.

Simplifying a bit:

$\max _{|p| \leq g} \int_{\Omega} f_{2} \operatorname{div} p-\frac{\theta}{2}(\operatorname{div} p)^{2} d x$.

Variations of Energy in (35) with respect to the vector field $p$ give:

$\int_{\Omega}\left(-\nabla f_{2}+\theta \nabla \operatorname{div} p\right) \cdot \delta p d x$

Along with the point-wise constraint $|p|^{2}-g^{2} \leq 0$, one gets the optimality condition:

$-\nabla\left(\theta \operatorname{div} p-f_{2}\right)+\lambda(x) p=0$,

where the Lagrange multiplier $\lambda(x) \geq 0$ for all $x$. As Chambolle shows in [19], it can be determined and eliminated as follows: If the constraint is not active at a point $x$, i.e., if $|p(x)|^{2}<g^{2}(x)$, then $\lambda(x)=0$. Otherwise, if the constraint is active at a point $x$, i.e., if $|p(x)|^{2}=g^{2}(x)$, then

$\left|\nabla\left(\theta \operatorname{div} p-f_{2}\right)\right|^{2}-\lambda^{2} g^{2}(x)=0$,

which leads to the conclusion that in either case, the value of $\lambda(x)$ is given by:

$\lambda=\frac{1}{g(x)}\left|\nabla\left(\theta \operatorname{div} p-f_{2}\right)\right|$.

Substituting (37) into (36) gives:

$-\nabla\left(\theta \operatorname{div} p-f_{2}\right)+\frac{1}{g(x)}\left|\nabla\left(\theta \operatorname{div} p-f_{2}\right)\right| p=0$.

We can use a semi-implicit gradient descent algorithm, as proposed by Chambolle in [19], to solve (38):

$p^{n+1}=\frac{p^{n}+\delta t \nabla\left(\operatorname{div} p^{n}-f_{2} / \theta\right)}{1+\frac{\delta t}{g(x)}\left|\nabla\left(\operatorname{div} p^{n}-f_{2} / \theta\right)\right|}$.

Hence, the difference of the iteration process (and the whole calculation) from the standard work of Chambolle is the appearance of the factor $g(x)$ in the denominator.

Proof of Proposition 2 The proof is the same as the one proposed in $[21,41]$. It is a simple $1-\mathrm{D}$ minimization problem, 
since all the equations are independent, and the computation is straightforward.

Proof of Theorem 2 The proof is in [13], based on [29, 37, 38], by replacing the TV-norm by the weighted TV-norm introduced in Definition 1. Like in the proof of Theorem 1, it basically consists of expressing Energy (17) in terms of the level sets of $u$ and $f$ and solving a geometric problem point-wise in $\mu$.

Proof of Theorem 3 The proof is in [13] with the weighted TV-norm replacing the TV-norm.

Proof of Proposition 4 Assume that $\mu$ has been chosen large enough (compared to $\lambda$ and $\|f\|_{L^{\infty}}$ ) so that exact penalty formulation works. We now consider the $v$-minimization:

$\min _{v} \int_{\Omega} \lambda r_{1}\left(x, c_{1}, c_{2}\right) v+\alpha v(v)+\frac{1}{2 \theta}(v-u)^{2} d x$.

The following claim helps with this step:

Claim If $u(x) \in[0,1]$ for all $x$, then so is $v(x)$ after the $v$ minimization. Conversely, if $v(x) \in[0,1]$ for all $x$, then so is $u(x)$ after the $u$-minimization.

This claim allows us to ignore the $v(v)$ term: Its presence in the energy is equivalent to cutting off $v(x)$ at 0 and at 1 (similar to what happens in [21]). On the other hand, if $v(x) \in[0,1]$, then the point-wise optimal $v(x)$ is found as:

$\theta \lambda r_{1}+(v-u)=0 \Rightarrow v(x)=u(x)-\theta \lambda r_{1}\left(x, c_{1}, c_{2}\right)$.

Thus, the $v$-minimization can be achieved through the following update:

$v=\min \left\{\max \left\{u(x)-\theta \lambda r_{1}\left(x, c_{1}, c_{2}\right), 0\right\}, 1\right\}$.

\section{References}

1. Kass, M., Witkin, A., Terzopoulos, D.: Snakes: active contour models. Int. J. Comput. Vis. 1(14), 321-331 (1998)

2. Malladi, R., Kimmel, R., Adalsteinsson, D., Sapiro, G., Caselles, V., Sethian, J.: A geometric approach to segmentation and analysis of 3D medical images. In: Mathematical Methods, Biomedical Image Analysis Workshop (1996)

3. Yezzi, A., Kichenassamy, S., Kumar, A., Olver, P., Tannenbaum, A.: A geometric snake model for segmentation of medical imagery. IEEE Trans. Med. Imaging 16(2), 199-209 (1997)

4. Jonasson, L., Bresson, X., Hagmann, P., Cuisenaire, O., Meuli, R., Thiran, J.: White matter fiber tract segmentation in DT-MRI using geometric flows. Med. Image Anal. 9(3), 223-236 (2005)

5. Caselles, V., Kimmel, R., Sapiro, G.: Geodesic active contours. Int. J. Comput. Vis. 22(1), 61-79 (1997)

6. Kichenassamy, S., Kumar, A., Olver, P., Tannenbaum, A., Yezzi, A.: Conformal curvature flows: from phase transitions to active vision. Arch. Ration. Mech. Anal. 134, 275-301 (1996)
7. Crandall, M., Ishii, H., Lions, P.: Users' guide to viscosity solutions of second order partial differential equations. Bull. Am. Math. Soc. 27(1), 1-69 (1992)

8. Osher, S., Sethian, J.: Fronts propagating with curvaturedependent speed: Algorithms based on Hamilton-Jacobi formulations. J. Comput. Phys. 79(1), 12-49 (1988)

9. Osher, S.: Level set methods. In: Osher, S., Paragios, N. (eds.) Geometric Level Set Methods in Imaging, Vision and Graphics, pp.3-20. Springer, New York (2003)

10. Sethian, J.: Level set methods and fast marching methods: evolving interfaces. In: Computational Geometry, Fluid Mechanics, Computer Vision and Material Sciences. Cambridge University Press, Cambridge (1999)

11. Osher, S., Fedkiw, R.: Level Set Methods and Dynamic Implicit Surfaces. Springer, New York (2003)

12. Jiang, G.-S., Peng, D.: Weighted ENO schemes for HamiltonJacobi equations. J. Sci. Comput. 21(6), 2126-2143 (1999)

13. Chan, T., Esedoḡlu, S., Nikolova, M.: Algorithms for finding global minimizers of image segmentation and denoising models, UCLA CAM Report 04-54 (2004)

14. Chan, T., Vese, L.: Active contours without edges. IEEE Trans. Image Process. 10(2), 266-277 (2001)

15. Rudin, L.I., Osher, S., Fatemi, E.: Nonlinear total variation based noise removal algorithms. Phys. D 60(1-4), 259-268 (1992)

16. Mumford, D., Shah, J.: Optimal approximations of piecewise smooth functions and associated variational problems. Commun. Pure Appl. Math. 42, 577-685 (1989)

17. Chan, T., Golub, G., Mulet, P.: A nonlinear primal-dual method for total variation-based image restoration. SIAM J. Sci. Comput. 20(6), 1964-1977 (1999)

18. Carter, J.: Dual methods for total variation-based image restoration. Ph.D. thesis, UCLA (2001)

19. Chambolle, A.: An algorithm for total variation minimization and applications. J. Math. Imaging Vis. 20(1-2), 89-97 (2004)

20. Aujol, J.-F., Chambolle, A.: Dual norms and image decomposition models. Int. J. Comput. Vis. 63(1), 85-104 (2005)

21. Aujol, J.-F., Gilboa, G., Chan, T., Osher, S.: Structure-texture image decomposition-modeling, algorithms, and parameter selection. Int. J. Comput. Vis. 67(1), 111-136 (2006)

22. Alliney, S.: Digital filters as absolute norm regularizers. IEEE Trans. Signal Process. 40(6), 1548-1562 (1992)

23. Alliney, S.: Recursive median filters of increasing order: a variational approach. IEEE Trans. Signal Process. 44(6), 1346-1354 (1996)

24. Alliney, S.: A property of the minimum vectors of a regularizing functional defined by means of the absolute norm. IEEE Trans. Signal Process. 45(4), 913-917 (1997)

25. Cheon, E., Paranjpye, A.: Noise removal by total variation minimization. UCLA MATH 199 project report, adviser. L. Vese (2002)

26. Nikolova, M.: Minimizers of cost-functions involving nonsmooth data-fidelity terms. SIAM J. Numer. Anal. 40(3), 965-994 (2002)

27. Alliney, S.: A variational approach to remove outliers and impulse noise. J. Math. Imaging Vis. 20(12), 99-120 (2004)

28. Alliney, S.: Weakly constrained minimization. Application to the estimation of images and signals involving constant regions. J. Math. Imaging Vis. 21(2), 155-175 (2004)

29. Chan, T., Esedoḡlu, S.: Aspects of total varation regularized $L^{1}$ function approximation. UCLA CAM Report 04-07 (2004)

30. Meyer, Y.: Oscillating patterns in image processing and in some nonlinear evolution equations. The Fifteenth Dean Jacquelines B. Lewis Memorial Lectures (2001)

31. Vese, L., Osher, S.: Modeling textures with total variation minimization and oscillating patterns. J. Sci. Comput. 19, 553-572 (2003) 
32. Bresson, X., Esedoḡlu, S., Vandergheynst, P., Thiran, J.-P., Osher, S.: Global minimizers of the active Contour/Snake model. UCLA CAM Report 05-04 (2005)

33. Vese, L., Chan, T.: A multiphase level set framework for image segmentation using the Mumford and Shah model. Int. J. Comput. Vis. 50(3), 271-293 (2002)

34. Cohen, L., Bardinet, E., Ayache, N.: Surface reconstruction using active contour models. In: SPIE International Symposium on Optics, Imaging and Instrumentation (1993)

35. Cohen, L., Kimmel, R.: Global minimum for active contour models: A minimal path approach. Int. J. Comput. Vis. 24(1), 57-78 (1997)

36. Appleton, B., Talbot, H.: Globally optimal geodesic active contours. J. Math. Imaging Vis. 23(1), 67-86 (2005)

37. Strang, G.: $L^{1}$ and $L^{\infty}$ approximation of vector fields in the plane. In: Nonlinear Partial Differential Equations in Applied Science, pp. 273-288. Kinokuniya, Tokyo (1982)
38. Strang, G.: Maximal flow through a domain. Math. Program. 26(2), 123-143 (1983)

39. Gomes, J., Faugeras, O.: Reconciling distance functions and level sets. J. Vis. Commun. Image Represent. 11, 209-223 (2000)

40. Leung, S., Osher, S.: Global minimization of the active contour model with TV-inpainting and two-phase denoising. In: Variational, Geometric, and Level Set Methods in Computer Vision (VLSM). Lecture Notes in Computer Science, vol. 3752, pp. 149160. Springer, New York (2005)

41. Chambolle, A., Vore, R.D., Lee, N., Lucier, B.: Nonlinear wavelet image processing: Variational problems, compression, and noise removal through wavelet shrinkage. IEEE Trans. Image Process. 7(3), 319-335 (1998) 